\title{
Expansions With Coefficient Algorithms for Time Domain Responses of Skin Effect Lossy Coaxial Cables
}

\author{
D. R. Holt* \\ Institute for Basic Standards, National Bureau of Standards, Boulder, Colorado 80302
}

(March 3, 1970)

\begin{abstract}
Time domain step response expansions with coefficient algorithms are developed from the system function of a doubly terminated skin effect lossy coaxial transmission line. Three models of series impedance valid for (a) high frequency, (b) high and intermediate frequency, (c) high and low frequency, are incorporated into the system function. The system function is expanded via the method of polynomial expansions of analytic functions through generating relations and inverted term by term into the time domain through the inverse Laplace transform. Step responses for time domain reflectometry and transmission are developed, computed, and compared with experimental results.
\end{abstract}

Key words: Coefficient algorithms; expansions; inverse Laplace transform; reflectometry; skin effect; step response; system function; transmission.

\section{Introduction}

In the analysis of skin effect lossy transmission lines one encounters propagation, characteristic impedance, and reflection functions which depend on $\sqrt{s}$. For example, exp $\{-x \gamma(s)\}, Z_{0}(s)$, and $\rho(s)=\left(Z-Z_{0}(s)\right) /\left(Z+Z_{0}(s)\right)$ depend on terms like $K \sqrt{s}$ or $R+K \sqrt{s}$. Such functions appear in the study of pulse transmission in coaxial cables and integrated circuit transmission line structures, etc., see references $[1,2,3,4,5,6] .^{1}$

A method of solution in the class of problems for $R \neq 0$ is to affect rearrangements of the power series representing $\gamma(s), Z_{0}(s)$, and $\rho(s)$. A series representation for exp $(-x \gamma(s))$ is realized through the technique of polynomial expansions via generating relations. Term by term Laplace transform inversion is then possible and the resulting functional series with certain expansion coefficient algorithms is the time domain solution.

The time domain expansions which were obtained in this paper were specifically developed for the study of the pulse response of coaxial cables [6]. The conclusions of that study demonstrated that the planar skin effect transmission line series impedance models, the computational results, and the experimental data correlated quite well.

We are now ready to pose the principal problem: determine the pulse distortion of a doubly terminated coaxial cable with skin effect losses. A circuit model of the system appears in figure 1 for a transmission line of length $l$ with

(a) $Z_{s 0}(s)$, the source impedance;

(b) $Z_{r}(s)$, the receiving end impedance;

(c) $E_{1}(s)$, the voltage step generator;

(d) $E_{2}(s, 0)$ and $E_{2}(s, l)$, step responses at input and output transmission line terminals respectively.

*Electromagnetic Division, Pulse and Time Domain, NBS Boulder Laboratories, Boulder, Colorado 80302.

${ }^{1}$ Figures in brackets indicate the literature references at the end of this paper. 


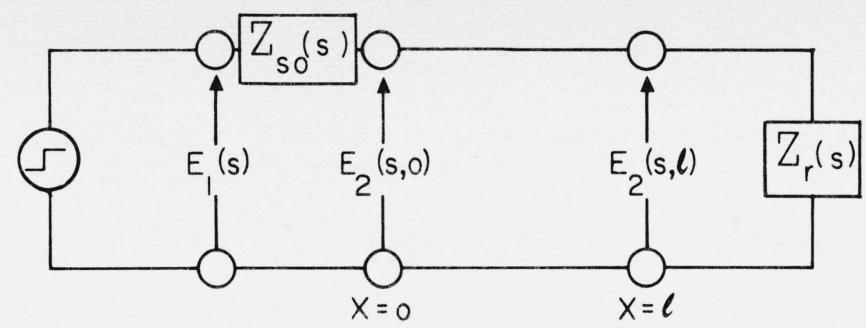

Figure 1. Circuit model of system function.

Proceeding to the system function [6]

$$
\frac{E_{2}(s, x)}{E_{1}(s)}=\frac{Z_{0}(s)}{Z_{0}(s)+Z_{s 0}(s)} \frac{\epsilon^{-x \gamma(s)}+\rho_{r}(s) \epsilon^{-(2 l-x) \gamma(s)}}{1-\rho_{s 0}(s) \rho_{r}(s) \epsilon^{-2 l \gamma(s)}}
$$

observe the voltage reflection function definitions

$$
\rho_{s 0}(s)=\frac{Z_{s 0}(s)-Z_{0}(s)}{Z_{s 0}(s)+Z_{0}(s)} \quad \text { and } \quad \rho_{r}(s)=\frac{Z_{r}(s)-Z_{0}(s)}{Z_{r}(s)+Z_{0}(s)}
$$

Now $\gamma(s)$ and $Z_{0}(s)$, propagation and characteristic impedance functions respectively, define as follows [6]:

$$
\begin{aligned}
\gamma(s) & =\sqrt{Z(s) Y(s)}, \\
Z_{0}(s) & =\sqrt{\frac{Z(s)}{Y(s)}}
\end{aligned}
$$

The functions $Z(s)$ and $Y(s)$ which are unit length transmission line parameters are known as series impedance and shunt admittance respectively. They define via the relations [6]

and

$$
Y(s)=C s
$$

$$
Z(s)=L s+K s^{1 / 2}+R
$$

where $K$ denotes the planar skin effect parameter.

The purpose of this paper is to obtain time domain step response expansions and expansion coefficient alogorithms from the system function (1.1) for three pairs of terminating impedances: (a) $Z_{s 0}(s)=0$ and $Z_{r}(s)=Z_{0}(s)$, (b) $Z_{s 0}(s)=\sqrt{L / C}$ and $Z_{r}(s)=+\infty$, (c) $Z_{s 0}(s)=Z_{r}(s)=\sqrt{L / C}$. We are interested in step responses at $x=0$ and/or $x=l$ for small and large time arguments.

In conjunction with the last remark observe that form (1.6) contains three models for series impedance [6] on selecting the proper value of $R$ : (a) $R=0$, high frequency; (b) $R=R^{\prime} / 4$, high and intermediate frequency; (c) $R=R^{\prime}$, high and low frequency. $R^{\prime}$ is the direct current (dc) resistance of the inner and outer conductors per unit length.

\section{Properties and Expansions of $\gamma(s)$ and $Z_{0}(s)$}

The following properties are important for extracting time domain expansions in later sections.

(a) Singularity property. Let $L_{1}$ be the defined $s$ domain of the inverse Laplace transform i.e., $-\pi<\arg s \leqslant \pi$. In view of (1.3), (1.4), (1.5) select the positive branch of $s^{1 / 2}$. Now keeping in mind that $L>0, K \geqslant 0, R \geqslant 0$ observe $L s+K s^{1 / 2}+R=0$ possesses roots $s_{0}^{1 / 2}$ and $s_{1}^{1 / 2}$ in the left hand plane. Therefore, arg $\mathrm{s}_{k} \notin L_{1}\left(k=0\right.$ or 1 ) and $\gamma(s)$ (or $\left.Z_{0}(s)\right)$ has one branch point $s=0$. 
(b) Bound on $\left|\epsilon^{-l \gamma(s)}\right|$. We want to show that $\left|\epsilon^{-l \gamma(s)}\right|<1$ for all interior points in the region comprised of the right-half plane (rhp). According to Titchmarsh [7] the maximum-modulus theorem is valid for either branch of $\exp \{-l \gamma(s)\}$ provided $|\exp \{-l \gamma(s)\}|$ is single valued at the origin. To see this is true let

$$
\gamma(\delta+j \omega)=|\gamma(\delta+j \omega)| \epsilon^{-j \varphi(\delta, \omega)} .
$$

Then clearly $\exp (-|\gamma(\delta+j \omega)| \cos \varphi(\delta, \omega)) \rightarrow \exp (0)=1$ independently of the path. Now on the rhp boundary $\left|e^{-l \gamma(s)}\right|$ has a maximum equal to 1 and a mimimum equal to 0 . Therefore, $\left|e^{-l \gamma(s)}\right|<1$ for $\operatorname{Re} s>0$.

(c) Lower bound on $\operatorname{Re} Z_{0}(s)$. Let $Z_{0}(s)=\left|Z_{0}(s)\right| \epsilon^{j \varphi_{0}(s)}$ and observe from (1.3), (1.4) and (b) above that $-\pi / 2 \leqslant \varphi(\delta, \omega) \leqslant \pi / 2$ implies $-\pi / 2 \leqslant \varphi_{0}(\delta, \omega) \leqslant \pi / 2$. Hence, $\operatorname{Re} Z_{0}(\delta+j \omega)=\mid Z_{0}(\delta$ $+j \omega) \mid \cos \varphi_{0}(\delta, \omega) \geqslant 0$.

(d) Expansions. It is convenient to set $s / \sqrt{L C}=\sigma$ in obtaining a binomial expansion of $\gamma(s)$. Then

$$
\begin{gathered}
l \gamma(s) \equiv \hat{\gamma}(\sigma)=\sigma\left\{1+\sum_{n=0}^{\infty}(-1)^{n} c_{n}\left\{\frac{\beta_{0}+\beta_{2} \sigma^{1 / 2}}{\sigma}\right\}^{n+1}\right\}, \\
c_{n}=\left|\frac{1}{2}\left(-\frac{1}{2}\right) \ldots\left(\frac{1}{2}-n\right)\right| \frac{1}{(n+1) !}, \mid \\
\beta_{0}=\frac{R}{L} l \sqrt{L C}, \quad \beta_{2}=\frac{K}{L} l^{1 / 2}(L C)^{1 / 4} .
\end{gathered}
$$

Implementing the binomial theorem in (2.1) produces

$$
\begin{aligned}
\hat{\gamma}(\sigma)= & \sigma\left\{1+c_{0} \sigma_{0}^{-1}\left\{d_{1,0}^{(1)}+d_{1,1}^{(1)} \sigma^{1 / 2}\right\}-c_{0} \sigma_{0}^{-2} \underset{0}{\left\{d_{2,0}^{(1)}+d_{2,1}^{(1)} \sigma^{1 / 2}\right.}\right. \\
& \left.+d_{2,2}^{(1)} \sigma^{2 / 2}\right\}+\ldots+(-1)^{n+m-1} c_{n+m-1} \sigma^{-2} \frac{(n+m)}{2} \sum_{k=0}^{n+m} d_{n+m, k}^{(1)} \sigma^{k / 2} \\
& \left.+(-1)^{n+m} c_{n+m} \sigma^{-\frac{2(n+m+1)}{2}} \sum_{k=0}^{n+m+1} d_{n+m+1, k}^{(1)} \sigma^{k / 2}+\ldots\right\}_{1}
\end{aligned}
$$

where

$$
d_{n, k}^{(1)}=\frac{n !}{(n-k) ! \mathrm{k} !} \beta_{0}^{n-k} \beta_{2}^{k}
$$

This series converges absolutely when $\infty<|s|<S_{2}=\left\{\frac{K}{2 L}+\sqrt{\frac{K^{2}}{4 L^{2}}}+\frac{R}{L}\right\}^{2}$ i.e., a proof of the bound appears in appendix A. Hence, rearrangement of the series (2.3) is realized by recognizing that $n+1$ terms contribute $\sigma^{\frac{-2 n}{2}}\left(\sigma^{\frac{-2 n+1}{2}}\right)$. We require that integral value of $k$ in (2.3) which cancels $\sigma^{-m}\left(\sigma^{-m-1 / 2}\right)$ and obtain the rearranged series

$$
\hat{\gamma}(\sigma)=\sigma\left\{1+\sum_{n=0}^{\infty} a_{1, n+1}^{(1)} \sigma^{-\frac{n+1}{2}}\right\}
$$

with coefficient algorithms

$$
\begin{gathered}
a_{1,2 n}^{(1)}=(-1)^{n-1} \sum_{m=0}^{n}(-1)^{m} c_{n+m-1} d_{n+m, 2 m}^{(1)}, \\
a_{1,2 n+1}^{(1,2)}=(-1)^{n} \sum_{m=0}^{n}(-1)^{m} c_{n+m} d_{n+m+1,2 m+1}^{(1)} .
\end{gathered}
$$


Similarly an expansion of the positive branch of $\gamma(s)$ for $0<|s| \leqslant S_{0}<\operatorname{minimum}\left(\frac{1}{2} \frac{R}{L} \frac{1 R^{2}}{4 K^{2}}\right)$ i.e., valid for small $|s|$, yields the series

$$
\gamma(s)=(R C)^{1 / 2} s^{1 / 2}\left\{1+\sum_{n=0}^{\infty}(-1)^{n} c_{n}\left\{\frac{K}{R} s^{1 / 2}+\frac{L}{R} s\right\}^{n+1}\right\}
$$

Proceeding as in (2.3) we find the rearrangement

$$
\gamma(s)=\sqrt{R C} s^{1 / 2}\left\{1+\sum_{n=0}^{\infty} a_{1, n+1}^{(2)} s^{\frac{n+1}{2}}\right\},
$$

with coefficients algorithms ${ }^{2}$

$$
\begin{gathered}
a_{1,2 n}^{(2)}=(-1)^{n-1} \sum_{m=0}^{n}(-1)^{m} c_{n+m-1} d_{n+m, n-m}^{(2)}, \\
a_{1,2 n+1}^{(2)}=(-1)^{n} \sum_{m=0}^{n}(-1)^{m} c_{n+m} d_{n+m+1, n-m}^{(2)}, \\
d_{n, k}^{(2)}=\frac{n !}{(n-k) ! k !} \eta_{0}^{n-k} \eta_{2}^{k}, \quad \eta_{0}=\frac{K}{R}, \quad \eta_{2}=\frac{L}{R}
\end{gathered}
$$

\section{General Method for Time Domain Solutions of the System Function}

The following two inverse Laplace transforms are well known [8]:

$$
\begin{gathered}
\mathscr{L}^{-1}\left\{\frac{\epsilon^{-k_{1} \sigma^{1 / 2}}}{\sigma^{\frac{n}{2}+1}}\right\}=(4 \tau)^{n / 2} I^{n} \operatorname{erfc}\left(\frac{k_{1}}{2} \tau^{-1 / 2}\right) \quad(n=0,1, \ldots), \\
\mathscr{L}^{-1}\left\{s^{\frac{n-1}{2}} \epsilon^{-k_{2}} s^{1 / 2}\right\}=\frac{\exp \left\{-\frac{k_{2}^{2}}{4 t}\right\}}{2^{n} \sqrt{\pi t^{n+1}}} H_{n}\left(\frac{k_{2}}{2 t^{1 / 2}}\right) \quad(n=0,1, \ldots) .
\end{gathered}
$$

Note $k_{1}$ and $k_{2}$ are positive; $I^{n} \operatorname{erfc} u\left(u=k / 2 t^{1 / 2}\right)$ is the $n$th repeated integral of erfc $x$ and possesses the recursion relation [9]

$$
I^{n} \operatorname{erfc} u=\int_{u}^{\infty} I^{n-1} \operatorname{erfc} x d x
$$

where $I^{0}$ erfc $x=\operatorname{erfc} x$. The notation $H_{n}(u)$ denotes Hermite polynomials of degree $n$.

With (1.1), (3.1), (3.2) in mind we require a method of expanding $A(w) \exp (\alpha g(w))$ such that $\alpha=\alpha(x)$, and $g(w)$ yields a Taylor expansion in $\sigma^{-1 / 2}$ (or $\left.s^{1 / 2}\right)$. From Boas and Buck [10] the technique of polynomial expansions of analytic functions through generating relations suffices in view of the following definition:

A set of polynomials has a Sheffer representation if it is generated by the formal relation

$$
A(w) \exp (\alpha g(w))=\sum_{n=0}^{\infty} P_{n}(\alpha) w^{n}
$$

\footnotetext{
${ }^{2}$ Superscript values of 1 and $k \geqslant 2$ correspond to high and low frequency expansions respectively on all coefficients.
} 
where

$$
\left.\begin{array}{ll}
A(w)=\sum_{n=0}^{\infty} A_{n} w^{n}, & A_{0} \neq 0, \\
g(w)=\sum_{n=0}^{\infty} g_{n} w^{n}, & g_{1} \neq 0
\end{array}\right\}
$$

If $g_{1}=0$ and $g_{n} \neq 0(n=2,3, \ldots)$ we define $P_{n}(\alpha)$ to be a modified Sheffer polynomial. This definition clearly indicates Sheffer polynomials are rather general since they are functions of the expansion coefficients in $A(w), g(w)$, exp $(u)$. The mechanics of obtaining $P_{n}(\alpha)$ are taken up in section 4 .

The application of (3.3) to (1.1) requires a hierarchy of coefficient algorithms such that recursive properties hold within and between algorithms. To clarify this point note the $d$ coefficients of (2.5) require recursivity within (2.5) to determine the " $a$ " coefficients while application of (3.3) to exp $(-\hat{\gamma}(\sigma))$ yields at least recursivity between a new algorithm and (2.5).

\section{Ideal Step Response}

A very simple pair of impedance values for (1.1), $Z_{r}(s)=Z_{0}(s)$ and $Z_{s 0}(s)=0$, leads to the $\sigma$ domain ideal step response

$$
\hat{E}_{2}(\sigma, l)=\hat{E} \frac{\epsilon^{-\hat{\gamma}(\sigma)}}{\sigma}, \quad \hat{E}=E l \sqrt{L C} .
$$

By virtue of (3.3) this expression becomes

$$
\begin{gathered}
\hat{E}_{2}(\sigma, l)=\hat{E} \epsilon^{-a_{1,2}^{(1)}} \frac{\exp \left\{-\left(\sigma+a_{1,2}^{(1)} \sigma^{1 / 2}\right\}\right.}{\sigma} \hat{T}_{1}(\sigma, l), \\
\hat{T}_{1}(\sigma, l)=\exp \left\{+\sum_{n=0}^{\infty} g_{n}^{(1)} \sigma^{-\left(\frac{n+1}{2}\right)}\right\},
\end{gathered}
$$

where $g_{n-3}^{(1)}=-a_{1, n}^{(1)}(n \geqslant 3)$ and (4.3) is a member of (3.3). According to Knopp [11] the main rearrangement theorem applies to (4.3) since

$$
\sum_{n=0}^{\infty}\left|g_{n}^{(1)} \sigma^{-\left(\frac{n+1}{2}\right)}\right| \leqslant M<\infty
$$

i.e., (2.4) is absolutely convergent. Hence, proceeding to a tractable expansion of (4.3) obtains the rearrangement

$$
\left\{\sum_{n=0}^{\infty} g_{n}^{(1)} \sigma^{-\left(\frac{n+1}{2}\right)}\right\}^{k}=\sigma^{-k / 2} \sum_{n=0}^{\infty} \mathrm{G}_{k, n, 1}^{(1)} \sigma^{-n / 2}
$$

where all $G$ coefficients ${ }^{3}$ define [12] through the recursion

$$
G_{k, n, 1}^{(1)}=\frac{1}{n g_{0}^{(1)}} \sum_{p=1}^{n}\{p(k+1)-n\} g_{p}^{(1)} G_{k, n-p, 1}^{(1)}
$$

${ }^{3}$ The third subscript of $G$ means its integer value is a multiplier of each $g$ coefficient on the right-hand side of the definition equation (4.5a). It corresponds to the number of reflections (or transmissions) which the input step voltage experiences. 
with initial values ${ }^{4}$

$$
G_{0,0,1}^{(1)}=1, \quad G_{k, 0,1}^{(1)}=\left(g_{0}^{(1)}\right)^{k}, \quad G_{1, k, 1}^{(1)}=g_{k}^{(1)} .
$$

From (4.3), (4.4), and (4.5) we obtain a double power series which convolutes with respect to subscripts and yields

$$
\begin{aligned}
\hat{T}_{1}(\sigma, l) & =\sum_{n=0}^{\infty} b_{n, 1}^{(1)} \sigma^{-n / 2}, \\
b_{n, 1}^{(1)} & =\sum_{k=1}^{n} \frac{G_{k, n-k, 1}^{(1)},}{k !} b_{0,1}^{(1)}=1,5
\end{aligned}
$$

such that all coefficients $b_{n, 1}^{(1)}$ are Sheffer polynomials with argument $x=l$.

Now following Doetsch [13] term by term inversion of (4.2) is permissible. Hence, in view of (3.1), (4.2), and (4.6) the time domain expression becomes

$$
\hat{e}_{2}(\tau, l)=E \epsilon^{-a_{1,2}^{(1)}} \sum_{n=0}^{\infty} b_{n, 1}^{(1)}\{4(\tau-1)\}^{n / 2} I^{n} \operatorname{erfc}\left\{\frac{a_{1,1}^{(1)}}{2}(\tau-1)^{-1 / 2}\right\}
$$

To determine the $\tau$ interval of uniform convergence observe that $\sum_{n=0}^{\infty}\left|b_{n, 1}^{(1)}\right| S_{4}^{-n / 2}\left(S_{4}>S_{2} l \sqrt{L C}\right)$ is a convergent series. If we require term by term dominance of the expansion in (4.8) the inequality

$$
\{4(\tau-1)\}^{n / 2} I^{n} \operatorname{erfc}\left\{\frac{a_{1,1}^{(1)}}{2}(\tau-1)^{-1 / 2}\right\} \leqslant S_{4}^{n / 2}
$$

determines the interval $1 \leqslant \tau \leqslant \tau_{0}$ where $\tau_{0}$ is that value of $\tau$ which makes (4.9) an equality.

To compute the step response for large time arguments revert to (2.7) and consider the $s$ domain expansion with coefficients $g_{m}^{(2)}=-l \sqrt{R C} a_{1, m+1}^{(2)}(m=0,1$. . .). Then

$$
E_{2}(s, l)=E \frac{\epsilon^{-l \gamma(s)}}{s}=E \frac{\epsilon^{-l \sqrt{R C} s^{1 / 2}}}{s} \exp \left\{\sum_{m=0}^{\infty} g_{m}^{(2)} s^{\frac{m+2}{2}}\right\} .
$$

For $0<|s| \leqslant S_{0}$ we use the argument supporting (4.6) to obtain

$$
\begin{aligned}
E_{2}(s, l) & =\frac{\epsilon}{s}^{-l \sqrt{R C} s^{1 / 2}} \sum_{n=0}^{\infty} b_{n, 1}^{(2)} s^{n / 2}, \\
b_{n, 1}^{(2)} & =\sum_{k=1}^{[n / 2]} \frac{G_{k, n-k, 1}^{(2)}}{k !}, \quad b_{1,1}^{(2)}=0,
\end{aligned}
$$

where $[n / 2]$ denotes the integer function. The coefficients $b_{n, 1}^{(2)}$ are modified Sheffer polynomials with argument $x=l$.

Now inversion of (4.11) through use of (4.3) and (4.4) carries $|s|$ beyond $S_{0}$. Hence, according to Doetsch [14], we can expect the time domain expansion to be asymptotic. Indeed, this turns out to be true under rearrangement of (4.11) into odd and even numbered terms from a proof in appendix B. Hence, the conclusion contains the form

\footnotetext{
${ }^{4}$ Initial values of $G$ coefficients assume the same form as $(4.5 \mathrm{~b})$ when superscripts are changed.

${ }^{5}$ We define $b_{0, n}^{(k)} \equiv 1$, independent of $n$ and $k$.
} 


$$
e_{2}(t, l) \sim E \operatorname{erfc}\left(\frac{l \sqrt{R C}}{2 t^{1 / 2}}\right)+\frac{E}{\pi^{1 / 2}} \exp \left\{\frac{-l^{2} R C}{4 t}\right\} \times\left\langle\sum_{n=1}^{\infty} b_{n, 1}^{(2)} \frac{H_{n-1}\left(\frac{l \sqrt{R C}}{2 t^{1 / 2}}\right)}{2^{n-1} t^{n / 2}}\right\rangle
$$

where the operator \langle\rangle denotes rearrangement with respect to $n$.

\section{Open Circuit Step Response}

To handle the open circuit case revert to $(1.1)$, set $Z_{s 0}(s)=\sqrt{L / C}$ and $Z_{r}(s)=+\infty$, and observe the input terminal step response

$$
E_{2}(s, 0)=E_{1}(s) \frac{Z_{0}(s)}{Z_{0}(s)+\sqrt{L / C} 1} \cdot \frac{1+\epsilon^{-2 l \gamma(s)}}{-\rho_{s 0}(s) \epsilon^{-2 l \gamma(s)}} .
$$

At this point it is worthwhile to investigate the possibility of expressing the $\tau$ time domain solution of (5.1) as a sequence of reflections. This corresponds to expressing $\left(1-\rho_{s 0}(s) \epsilon^{-2 l \gamma(s)}\right)^{-1}$ as a geometric series. The condition $\left|\rho_{s 0}(s) \epsilon^{-2 l \gamma(s)}\right|<1$ is satisfied in view of $3 \mathrm{~b}$ and $3 \mathrm{c}$. We remark that it is also quite easy to calculate an upper bound for $\left|\rho_{s 0}(s)\right|$ by Stannard's method [15].

Applying the geometric expansion to (5.1) yields

$$
\hat{E}_{2}(\sigma, 0)=\hat{E}_{1}(\sigma) \frac{\hat{Z}_{0}(\sigma)}{\hat{Z}_{0}(\sigma)+\sqrt{L / C}} \sum_{n=0}^{\infty} \hat{\rho}_{s 0}^{n}(\sigma)\left\{\epsilon^{-2 n \hat{\gamma}(\sigma)}+\epsilon^{-2(n+1) \hat{\gamma}(\sigma)}\right\}
$$

Following the expansion technique for $\hat{\gamma}(\sigma)$ obtains the "unreflected term" representation

$$
\begin{gathered}
\hat{E}_{2,0}(\sigma, 0) \equiv \frac{\hat{Z}_{0}(\sigma)}{\hat{Z}_{0}(\sigma)+\sqrt{L / C}}=\frac{1}{2}+\sum_{n=0}^{\infty} a_{2, n+1}^{(1)} \sigma^{-\left(\frac{n+1}{2}\right)}, \\
a_{2,2 n}^{(1)}=(-1)^{n-1} \sum_{k=0}^{n}(-1)^{k} c_{n+k} d_{n+k, 2 k}^{(1)}, \\
a_{2,2 n+1}^{(1)}=(-1)^{n} \sum_{k=0}^{n}(-1)^{k} c_{n+k+1} d_{n+k+1,2 k+1}^{(1)} .
\end{gathered}
$$

Integral powers of voltage reflection functions expand into the form

$$
\hat{\rho}_{s 0}^{n}(\sigma)=(-2)^{n} \sum_{m=0}^{\infty} A_{m+1, n-1}^{(1)} \sigma^{-\left(\frac{m+n}{2}\right)} \quad(n=1,2, \ldots)
$$

which defines itself according to the alternate recursion relations ${ }^{6}$

$$
A_{m+1,0}^{(1)} \equiv a_{2, m+1}^{(1)} \text { and } A_{m+1, n-1}^{(1)}=\sum_{k=0}^{m} a_{2, m+1-k}^{(1)} A_{k+1, n-2}^{(1)} .
$$

We expand the exponent functions of (5.2) into the form

$$
\epsilon^{-2 p \gamma(\sigma)}=\epsilon^{-2 p a_{1,2}^{(1)}} \exp \left\{-2 p\left\{\sigma+a_{1,1}^{(1)} \sigma^{1 / 2}\right\}\right\} \sum_{m=0}^{\infty} b_{m, 2 p}^{(1)} \sigma^{-m / 2}
$$

${ }^{6}$ Of course relations (4.5) and (5.5) give the same answer. However, their implementation points out one difference which we shall mention in section 7. 
such that $p=n, n+1$. Hence, from (5.3), (5.4), and (5.6) the $n$th term becomes

$$
\begin{aligned}
\hat{E}_{2, n}(\sigma, 0)=\hat{E}(-2)^{n}\left\{\sum_{p=n}^{n+1} \epsilon^{-2 p a_{1,2}^{(1)}} \exp \frac{\left\{-2 p\left\{\sigma+a_{1,1}^{(1)} \sigma^{1 / 2}\right\}\right\}}{0}\right. & \sigma \\
\times & \left\{\frac{1}{2} \sum_{m=0}^{\infty}\left\{\sum_{k=0}^{m} b_{m-k, 2 p}^{(1)} A_{k+1, n-1}^{(1)}\right\}_{0} \sigma^{-\left(\frac{m+n}{2}\right)}\right. \\
& \left.\left.+\left\{\sum_{k=0}^{m} b_{m-k, 2 p}^{(1)} A_{k+1, n}^{(1)}\right\} \sigma_{0}^{-\left(\frac{m+n+1}{2}\right)}\right\}\right\}_{2} \quad(n=0,1, \ldots) .
\end{aligned}
$$

The prime notation on sigma indicates the series $(5.3 \mathrm{a})$ replaces (5.7) at $p=0$ and, at $p=1, b_{m, 2}^{(1)}$ replaces the sum $\sum_{k=0}^{m} b_{m-k, 2}^{(1)} A_{k+1,-1}^{(1)}$.

Hence, the time domain response yields

$$
\begin{aligned}
& e_{2}(\tau, 0)=E \sum_{n=0}^{[\tau]}(-2)^{n}\left\{\sum _ { 2 } ^ { n + 1 } \sum ^ { - 2 p } \epsilon ^ { ( 1 , 2 ) } \left\{\frac{1}{2} \sum_{m=0}^{\infty}\left\{\sum_{k=0}^{m} b_{m-k, 2 p}^{(1)} A_{k+1, n-1}^{(1)}\right\}\right.\right.
\end{aligned}
$$

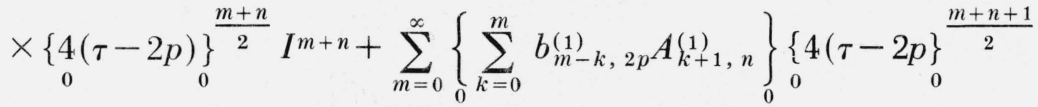

$$
\begin{aligned}
& \left.\left.\left.\times I^{m+n+1}\right\}_{1} \operatorname{erfc}\left\{p a_{1}^{(1)}(\tau-2 p)^{-1 / 2}\right\}\right\}_{1}\right\}
\end{aligned}
$$

and the term for $p=0$ inverts to the series

$$
\hat{e}_{2,0}(\tau, 0)=\frac{1}{2}+\sum_{m=0}^{\infty} a_{2, m+1}^{(1)} \frac{\tau^{(m+1) / 2}}{\Gamma\left(\frac{m+3}{2}\right)} .
$$

This series converges uniformly when

$$
\left(\tau^{m+1 / 2}\right) / \Gamma\left(\frac{m+1}{2}\right) \leqslant S_{4}^{-\frac{m+1}{2}}
$$

Clearly inequality (4.9) ( $n$ replaced by $m+n$ ) extends to cover (5.8).

To determine the time domain asymptotic expansion let

$$
\left.\frac{Z_{0}(s)}{Z_{0}(s)+\sqrt{\frac{L}{C}}}=\left\{1+\eta_{0} s^{1 / 2}+\eta_{2} s-\eta_{2}^{1 / 2} s^{1 / 2}\left\{1+\sum_{n=0}^{\infty} a_{1, n+1}^{(2)} s^{(n+1) / 2}\right\}\right\}_{0}\right\}_{1} \frac{1}{1+\eta_{0} s^{1 / 2}} .
$$

Now define a set of coefficients such that

$$
A_{0}^{(2)}=1, \quad A_{1}^{(2)}=\eta_{0}-\eta^{1 / 2}, \quad A_{2}^{(2)}=\eta_{2}-\eta_{2}^{1 / 2} a_{1,1}^{(2)}, \quad A_{n}^{(2)}=-\eta_{2}^{1 / 2} a_{1, n-1}^{(2)} \quad(n=3,4, \ldots) .
$$


Then (5.11) assumes the form

$$
\frac{Z_{0}(s)}{Z_{0}(s)+\sqrt{\frac{L}{C}}}=\frac{1}{1+\eta_{0} s^{1 / 2}} \sum_{n=0}^{\infty} A_{n}^{(2)} s^{n / 2}
$$

In a very similar manner we obtain the expansion

$$
\rho_{s 0}(s)=-\frac{1}{1+\eta_{0} s^{1 / 2}} \sum_{n=0}^{\infty} A_{n, 0}^{(3)} s^{n / 2}
$$

with coefficient definitions

$$
A_{0,0}^{(3)}=1, \quad A_{1,0}^{(3)}=\eta_{0}-2 \eta_{2}^{1 / 2}, \quad A_{2,0}^{(3)}=2\left\{\eta_{2}-\eta_{2}^{1 / 2} a_{1,1}^{(2)}\right\}, \quad A_{n, 0}^{(3)}=-2 \eta_{2}^{1 / 2} a_{1, n-1}^{(2)} \quad(n=3,4 \ldots . .
$$

For convenience redefining the coefficients of (2.7) yields

$$
a_{1,1}^{(3)}=-l \sqrt{R C}, \quad a_{1, n}^{(3)}=-l \sqrt{R C} a_{1, n-1}^{(2)} \quad(n=2,3, \ldots), \quad a_{1, n+1}^{(3)}=g_{n}^{(3)} \quad(n=0,1, \ldots)
$$

The exponential function of (5.1) has the expansion

$$
\epsilon^{-2 l \gamma(s)}=\sum_{k=0}^{\infty} 2^{k} \frac{s^{k / 2}}{k !} \times \sum_{m=0}^{\infty} G_{k, m, 1}^{(3)} s^{m / 2}=\sum_{n=0}^{\infty} b_{n, 2}^{(3)} s^{n / 2},
$$

with the coefficient algorithm

$$
b_{n, 2}^{(3)}=\sum_{k=1}^{n} \frac{G_{k, n-k, 2}^{(3)}}{k !} \quad(n \geqslant 2) .
$$

Combining expansions (5.13) and (5.14) obtains

$$
1-\rho_{s 0}(s) \epsilon^{-2 l \gamma(s)}=\frac{1}{1+\eta_{0} s^{1 / 2}} \sum_{n=0}^{\infty} M_{n}^{(2)} s^{n / 2},
$$

subject to coefficient algorithms

$$
\begin{aligned}
& M_{0}^{(2)}=2, \quad M_{1}^{(2)}=\eta_{0}+A_{1,0}^{(3)}+b_{1,2}^{(3)}, \\
& M_{n}^{(2)}=\sum_{k=0}^{n} A_{n-k, 0}^{(3)} b_{k, 2}^{(3)} \quad(n=2,3, \ldots)
\end{aligned}
$$

Expansions (5.12) through (5.15) constitute a scheme for the series solution of (5.1) by means of the two quotients

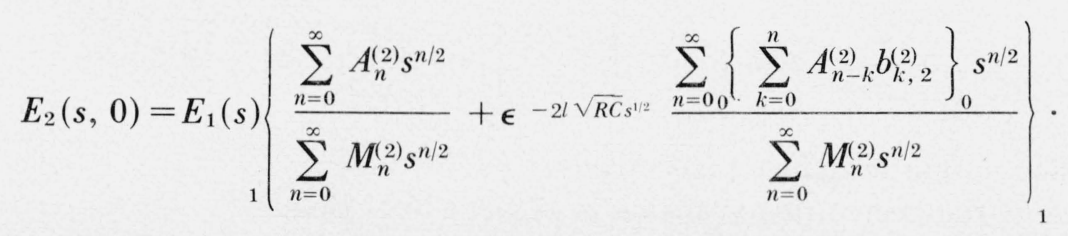


Since the power series above are analytic for $0<|s| \leqslant S_{0}$ we may perform division termwise to obtain

$$
E_{2}(s, 0)=E_{1}(s)\left\{\sum_{n=0}^{\infty} W_{n}^{(2)} s^{n / 2}+\epsilon^{-2 l \sqrt{R C} s^{1 / 2}} \sum_{n=0}^{\infty} W_{n}^{(3)} s^{n / 2}\right\}
$$

such that recursion formulas

$$
W_{n}^{(2)}=\frac{A_{n}^{(2)}-\sum_{k=0}^{n-1} M_{n+k}^{(2)} W_{k}^{(2)}}{2}
$$

and

$$
W_{n}^{(3)}=\frac{\sum_{k=0}^{n-1} A_{n-k}^{(2)} b_{k, 2}^{(2)}-\sum_{k=0}^{n-1} M_{n-k}^{(2)} W_{k}^{(3)}}{2} \quad(n=1,2, \ldots),
$$

with initial values $W_{0}^{(2)}=W_{0}^{(3)}=\frac{1}{2}$ enable all coefficient determinations. For a time domain expansion of (5.17) invoke the gamma function equation $\Gamma(z) \Gamma(1-z)=\pi \csc \pi z$ ( $z$ complex and nonintegral real) and observe

$$
\begin{aligned}
e_{2}(t, 0) \sim \frac{E}{2}+\frac{E}{\pi} \sum_{n=1}^{\infty}(-1)^{n-1} W_{2 n-1}^{(2)} \Gamma\left(\frac{2 n-1}{2}\right) t^{-\left(\frac{2 n-1}{2}\right)} \\
+\frac{E}{2} \operatorname{erfc}\left(\frac{l \sqrt{R C}}{2 t^{1 / 2}}\right)+E \epsilon^{-\frac{l^{2} R C}{4 t}}\left\langle\sum_{n=1}^{\infty} W_{n}^{(3)} \frac{H_{n-1}\left(\frac{l \sqrt{R C}}{2 t^{1 / 2}}\right)}{2^{n-1} \sqrt{\pi t^{n}}}\right\rangle
\end{aligned}
$$

as $t \rightarrow \infty$ is a complete asymptotic expansion.

The short circuit step response with impedance values $Z_{r}(s)=0$ and $Z_{s 0}(s)=\sqrt{\frac{L}{C}}$ is obtained from the basic $\sigma$ and $s$ domain expansions of this section.

\section{Time Domain Reflectometry and Transmission}

We select the impedance values $Z_{s 0}(s)=Z_{r}(s)=\sqrt{\frac{L}{C}}$ and require a solution of (1.1) for $0 \leqslant x \leqslant l$ and $0 \leqslant t \leqslant T<\infty$. Let $\hat{\rho}_{r}(\sigma)=\hat{\rho}_{s 0}(\sigma) \equiv \hat{\rho}(\sigma)$ and define three relations

$$
\left.\begin{array}{rl}
\alpha_{2 n}(x) & =2 \ln +x, \\
\alpha_{2 n+1}(x) & =2 l(n+1)-x, \\
\hat{\alpha}_{n}(x) & =\frac{1}{l} \alpha_{n}(x),(n=0,1,2 \ldots)
\end{array}\right\}
$$

which include two integer functions

$$
\hat{\alpha}_{n}(0)=2\left[\frac{n+1}{2}\right] \text { and } \hat{\alpha}_{n}(l)=2\left[\frac{n}{2}\right]+1
$$

at input and output terminals respectively.

Invoking the geometric expansion in (4.2) via (6.1) yields 


$$
\hat{E}_{2}(\sigma, x)=\hat{E}_{1}(\sigma) \frac{\hat{Z}_{0}(\sigma)}{\hat{Z}_{0}(\sigma)+\sqrt{\frac{L}{C}}} \sum_{n=0}^{\infty} \hat{\rho}^{n}(\sigma) \epsilon^{-\hat{\alpha}_{n}(x) \hat{\gamma}(\sigma)}
$$

To implement the rearranged series set

$$
T_{n}(\sigma, x)=\exp \left\{\hat{\alpha}_{n}(x) \sum_{m=0}^{\infty} g_{m}^{(1)} \sigma^{-\left(\frac{m+1}{2}\right)}\right\}
$$

Let the definition equation

$$
\left\{\alpha_{p}(x) \sum_{m=0}^{\infty} g_{m}^{(1)} \sigma^{-\left(\frac{m+1}{2}\right)}\right\}^{k}=\left\{\hat{\alpha}_{p}(x) \sigma^{-1 / 2}\right\}^{k} \sum_{m=0}^{\infty} J_{k, m}^{(1)} \sigma^{-m / 2}
$$

analogous to (4.4) apply such that all $J$ coefficients obey the relations (4.5). Then as in (5.5) we have the expansion

$$
T_{n}(\sigma, x)=\sum_{m=0}^{\infty} B_{m, n}^{(1)}(x) \sigma^{-m / 2}
$$

contingent on the definition

$$
B_{m, n}^{(1)}(x)=\sum_{k=1}^{m} \hat{\alpha}_{n}^{k}(x) J_{k, m-k}^{(1)} .
$$

The convergence of (6.5a) is uniform over $0 \leqslant x \leqslant l$ and $\infty \geqslant|\sigma| \geqslant S_{4}$. Since the inverse Laplace transform of $(6.2)$ is zero when $\tau=[T / / \sqrt{L C}]+1$ (integer function) we do not inquire about the convergence of $(6.2)$ with respect to $n$.

For $0 \leqslant x \leqslant l$ and $0 \leqslant n \leqslant \infty$, the $\sigma$ domain step response of (6.2) with support from (5.3), (5.4) and (6.5) becomes

$$
\begin{aligned}
\hat{E}_{2}(\sigma, x)= & \hat{E}_{1}(\sigma) \sum_{n=0}^{\infty} /(-2)^{n} \epsilon^{-\hat{\alpha}_{n}(x) a a_{1,2}^{(1,2}}\left\{\sum_{k=0}^{\infty}\left\{\frac{1}{2} \sum_{m=0}^{k} A_{k+1-m, n-1}^{(1)} B_{m, n}^{(1)}(x)\right\}\right. \\
& \left.\times \sigma^{-\left(\frac{k+n}{2}\right)}+\sum_{k=0}^{\infty}\left\{\sum_{m=0}^{k} A_{k+1-m, n}^{(1)} B_{m, n}^{(1)}(x)\right\} \sigma^{-\frac{k+n+1}{2}}\right\} \\
& \times \exp \left\{-\hat{\alpha}_{n}(x)\left(\sigma+a_{1,1}^{(1)} \sigma^{1 / 2}\right)\right\}
\end{aligned}
$$

where the polynomials of degree $k$ are Sheffer and the prime on sigma indicates two exceptions:

(a) Replace $\sum_{m=0}^{k} A_{k+1-m, n-1}^{(1)} B_{m, n}^{(1)}(x)$ with $B_{k, n}^{(1)}(x)$ at $n=0$,

(b) Replace (6.6) at $n=0$ and $x=0$ with (5.3a).

Hence, the time domain response yields

$$
\begin{aligned}
& \hat{e}_{2}(\tau, x)=E \sum_{n=0}^{[\tau]} /(-2)^{n} \epsilon^{-\hat{\alpha}_{n}(x) a_{1,2}^{(1)}}\left\{\sum_{1}^{\infty}\left\{\frac{1}{2} \sum_{m=0}^{k} A_{k+1-m, n-1}^{(1)} B_{m, n}^{(1)}(x)\right\}\right. \\
& \times\left\{4\left(\tau-\hat{\alpha}_{n}(x)\right)\right\}_{0}^{\frac{k+n}{2}} I^{k+n}+\sum_{k=0}^{\infty}\left\{\sum_{0}^{k} A_{k=0}^{(1)}{ }_{k+1-m, n} B_{m, n}^{(1)}(x)\right\}_{0}
\end{aligned}
$$

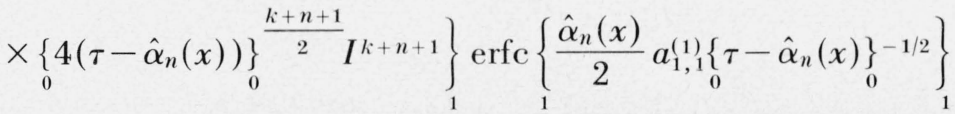


where (5.10) replaces $(6.7)$ at $n=0$ and $x=0$.

To determine the $\tau$ interval of uniform convergence invoke the inequality

$$
\left\{4\left(\tau-\hat{\alpha}_{n}(x)\right)\right\}^{\frac{k+p}{2}} I^{k+p} \operatorname{erfc}\left\{\frac{\hat{\alpha} n(x)}{2} a_{1,1}^{(1)}\left(\tau-\hat{\alpha}_{n}(x)\right)^{-1 / 2}\right\} \leqslant S_{4}^{-\frac{k+p}{2}}
$$

for $p=n, n+1$.

For large time intervals we first note that (5.13) and (5.14) furnish a starting point for the expansion

$$
1-\rho^{2}(s) \epsilon^{-2 l \gamma(s)}=\frac{s^{1 / 2}}{\left(1+\eta_{0} s^{1 / 2}\right)^{2}} \sum_{n=0}^{\infty} M_{n}^{(3)} s^{n / 2}
$$

such that $M_{n}^{(3)}$ coefficients define according to the rules:

$$
M_{0}^{(3)}=2 \eta_{0}-b_{1,2}^{(3)}-A_{1,1}^{(3)}, \quad M_{1}^{(3)}-\eta_{0}^{2}-\left\{A_{2,1}^{(3)}+b_{2,2}^{(3)}+A_{1,1}^{(3)} b_{1,2}^{(3)}\right\}, \quad M_{n}^{(3)}=-\sum_{k=0}^{n+1} A_{n+1-k, 1}^{(3)} b_{k, 2}^{(3)},
$$

where $A_{n, 1}^{(3)}=\sum_{k=0}^{n} A_{n-k, 0}^{(3)} A_{k, 0}^{(3)}$.

From (5-12) and (6.9) we have the product

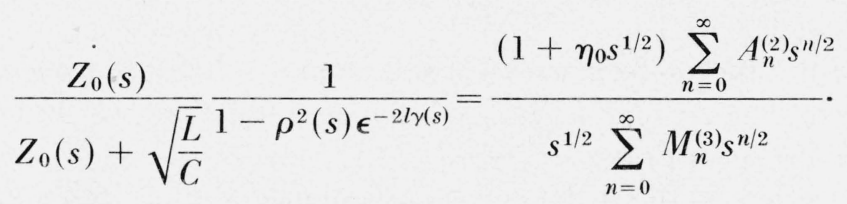

Following the methods of (6.3) and (6.4) produces

$$
\begin{aligned}
\epsilon^{-\alpha_{p}(x) \gamma(s)} & =\epsilon^{-\alpha_{p}(x) \sqrt{R C} s^{1 / 2}} \sum_{k=0}^{\infty} \frac{\left(\alpha_{p}(x) s\right)^{k}}{k !}\left\{\sum_{m=0}^{\infty} g_{m}^{(2)} s^{m / 2}\right\}^{k}, \\
& =\epsilon^{-\alpha p(x) \sqrt{R C} s^{1 / 2}} \sum_{n=0}^{\infty} B_{n, p}^{(2)}(x) s^{n / 2}
\end{aligned}
$$

contingent on the following definitions and properties:

$$
\begin{gathered}
B_{n, p}^{(2)}(x)=\sum_{k=1}^{[n / 2]} \frac{\alpha_{p}^{k}(x)}{k !} J_{k, n-2 k}^{(2)} \quad(n \geqslant 2), \\
B_{0, p}^{(2)}(x) \equiv 1, \quad B_{1, p}^{(2)}(x) \equiv 0, \quad B_{n, 0}^{(2)}(l) \equiv B_{n, 1}^{(2)}(l) .
\end{gathered}
$$

We define the equations

$$
\begin{aligned}
C_{n, 0}^{(2)}(x) & =\sum_{m=0}^{n} A_{n-m}^{(4)} B_{m, 0}^{(2)}(x) \quad(x>0), \\
C_{n, 0}^{(2)}(0) & =A_{n}^{(4)} \\
A_{0}^{(4)} & =1, \quad A_{n}^{(4)}=A_{n}^{(2)}+\eta_{0} A_{n-1}^{(2)},
\end{aligned}
$$




$$
\begin{gathered}
C_{n, 1}^{(2)}(x)=\sum_{k=0}^{n} \sum_{m=0}^{k} A_{n-k}^{(2)} A_{k-m, 0}^{(3)} B_{m, 1}^{(2)}(x), \\
N_{n, k}(x)=\frac{C_{n, k}^{(2)}(x)-\sum_{m=0}^{n-1} N_{m, k}(x) M_{n-m}^{(3)}}{M_{0}^{(3)}} \quad\left(\begin{array}{l}
n \geqslant 1 \\
x \geqslant 0
\end{array}\right) .
\end{gathered}
$$

From (6.11) and (6.12) three special cases condense into the forms

$$
\left.\begin{array}{l}
N_{0,0}(x)=N_{0,1}(x)=1 / M_{0}^{(3)}, \\
N_{1,0}(x)=\frac{A_{1}^{(4)}-M_{1}^{(3)} / M_{0}^{(3)}}{M_{0}^{(3)}}, \\
N_{1,1}(x)=\frac{A_{1}^{(2)}+A_{1,0}^{(3)}-M_{1}^{(3)} / M_{0}^{(3)}}{M_{0}^{(3)}}
\end{array}\right\} x \geqslant 0 .
$$

In the $s$ domain we have the representation

$$
E_{2}(s, x)=\sum_{k=0}^{1}(-1)^{k} \epsilon^{-\alpha} k^{(x)} \sqrt{\overline{R C}^{1 / 2}} \sum_{n=0}^{\infty} N_{n, k}(x) s^{(n-3) / 2}
$$

which converges uniformly in $x$ and $s$ over $0 \leqslant x \leqslant l$ and $0<\epsilon_{0} \leqslant|s| \leqslant S_{0}$. It is necessary to consider three positions on the line: (a) $x=0$, (b) $x=l$, (c) $0<x<l$. At $x=0$ the terms corresponding to $n=0$ on Laplace transform inversion contribute $t^{1 / 2}$, a term which cancels on using the series expansion of $I^{1}$ erfc ( ). Hence, observe the complete asymptotic expansion for time domain reflectometry (TDR).

$$
\begin{aligned}
e_{2}(t, 0) \sim E\left\{2 N_{0} l \sqrt{R C} \sum_{m=1}^{\infty} \frac{(-)^{m-1}\left\{\frac{2 l \sqrt{R C}}{t^{1 / 2}}\right\}^{m-1}}{m ! \Gamma\left(1+\frac{1-m}{2}\right)}+N_{1,0}(0) U(t)\right. \\
+\sum_{n=1}^{\infty}(-1)^{n-1} N_{2 n, 0}(0) \Gamma\left(\frac{2 n-1}{2}\right) t^{-\left(\frac{2 n-1}{2}\right)}-N_{1,1}(0) \operatorname{erfc}\left\{\frac{l \sqrt{R C}}{t^{1 / 2}}\right\} \\
\left.-\epsilon^{-\frac{l^{2} R C}{t}}\left\langle\sum_{n=2}^{\infty} N_{n, 1}(0) \frac{H_{n-2}\left\{\frac{l \sqrt{R C}}{t^{1 / 2}}\right\}}{2^{n-2} t^{(n-1) / 2}}\right\}\right\}
\end{aligned}
$$

as $t \rightarrow \infty$. In view of (6.12) and the coefficient definitions of section $5(6.15)$ at $t=\infty$ reduces to

$$
e_{2}(\infty, 0)=\frac{\sqrt{\frac{L}{C}}+l R}{2 \sqrt{\frac{L}{C}}+l R}
$$

the $\mathrm{d}$-c voltage divider equation.

Over $0<x<l$ we use the expansion of $I^{1}$ erfc ( ) again to cancel the term associated with $t^{1 / 2}$ and extract the complete asymptotic expansion 


$$
\begin{aligned}
e_{2}(t, x) \sim E\left\{N_{0} \sqrt{R C} \sum_{k=0}^{1}(-1)^{k} \alpha_{k}(x) \sum_{m=1}^{\infty} \frac{(-1)^{m-1}}{m ! \Gamma\left(1+\frac{1-m}{2}\right)}\left\{\frac{\alpha_{k}(x) \sqrt{R C}}{2 t^{1 / 2}}\right\}^{m-1}\right. \\
\left.+\sum_{k=0}^{1}(-1)^{k} N_{1, k} \operatorname{erfc}\left(\frac{\alpha_{k}(x) \sqrt{R C}}{2 t^{1 / 2}}\right)\right\} \\
\quad+E \sum_{k=0}^{1}(-1)^{k} \epsilon^{-\frac{\alpha_{k}^{2}(x) R C}{4 t}}\left\langle\sum_{n=2}^{\infty} N_{n, k}(x) \frac{\left.H_{n-2}\left\{\frac{\alpha_{k}(x) \sqrt{R C}}{2 t^{1 / 2}}\right\}\right\rangle}{2^{n-2} t^{(n-1) / 2}}\right\rangle
\end{aligned}
$$

as $t \rightarrow \infty$. In the case of time domain transmission (TDT) note $x=l$ means $\alpha_{0}(l)=\alpha_{1}(l)$ and, consequently the coefficient of $N_{0}$ vanishes. Also at $t=\infty$ and $x=l$ we obtain the d-c voltage divider expression

$$
e_{2}(\infty, l)=\frac{\sqrt{\frac{L}{C}}}{2 \sqrt{\frac{L}{C}}+l R} .
$$

\section{Realization of TDR and TDT Data}

\subsection{Theoretical and Experimental Results}

A transmission line with the following parameter values is illustrated: $L \doteq 1.184 \times 10^{-11} \mathrm{H} / \mathrm{m}$, $C \doteq 2.46 \times 10^{-7} \mathrm{~F} / \mathrm{m}, l \doteq 137.2 \mathrm{~m}(450 \mathrm{ft}), R^{\prime} \doteq 1.466 \times 10^{-2} \Omega / \mathrm{m}, K \sqrt{s} \doteq 3.35 \times 10^{-5} \sqrt{s} \Omega / \mathrm{m}$. Three curves of each figure correspond to three models of $Z(s)$ (sec. 1). Each theoretical curve approximates experimental data best over a certain time interval and the relative error between the two sets of data (taking either set as correct) over that time interval is realized through the following example:

\section{TDT Data}

$$
\left.\begin{array}{lllll}
t \text { (microseconds) } & 1 & 2 & 3 & 4 \\
\% \text { error } & 2 & 1 & 1 & 2
\end{array}\right\} Z(s)=\frac{R}{4}+K \sqrt{s}+L s .
$$

In view of a 3 percent uncertainty of the experimental system [6], a maximal 2 percent error for the appropriate $Z(s)$ in the data of the figures below is optimal. Experimental data points for $t$ less than $10 \mathrm{~ns}$ are not reliable [6].

\subsection{Computational Observations}

Computer programs were written on a CDC 3800 digital computer in Fortran language to realize theoretical TDR and TDT data. We mention certain considerations of the computational procedure for (6.7) by starting with $c$ and $d^{(1)}$ coefficients of (2.5a). Their product possesses recursions

where

$$
F_{n+1,0}^{(1)}=\frac{n-\frac{1}{2}}{n+1} \beta_{0} F_{n, 0}^{(1)}, \quad F_{n, k+1}^{(1)}=\frac{\left(n+k-\frac{1}{2}\right)(n-k)}{(2 k+1)(2 k+2)} \frac{\beta_{2}^{2}}{\beta_{0}} F_{n, k}^{(1)}
$$

$$
F_{n, k}^{(1)}=c_{n+k-1} d_{n+k, 2 k}^{(1)} \quad(0 \leqslant k \leqslant n \text { and } n=1,2, \ldots) .
$$




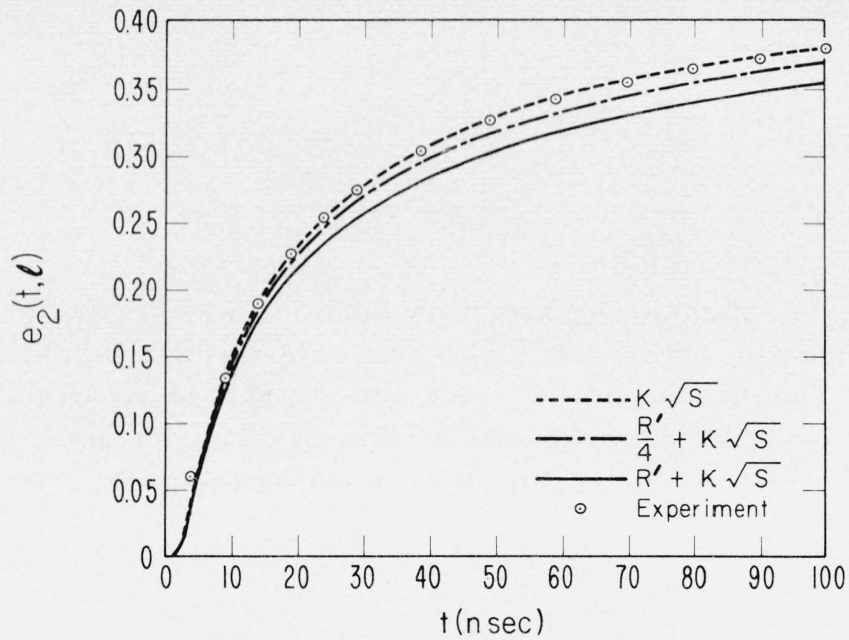

FIGURE 2. Step response at receiving end with one unit of time delay $(\mathrm{I} \sqrt{\mathrm{LC}})$ removed.

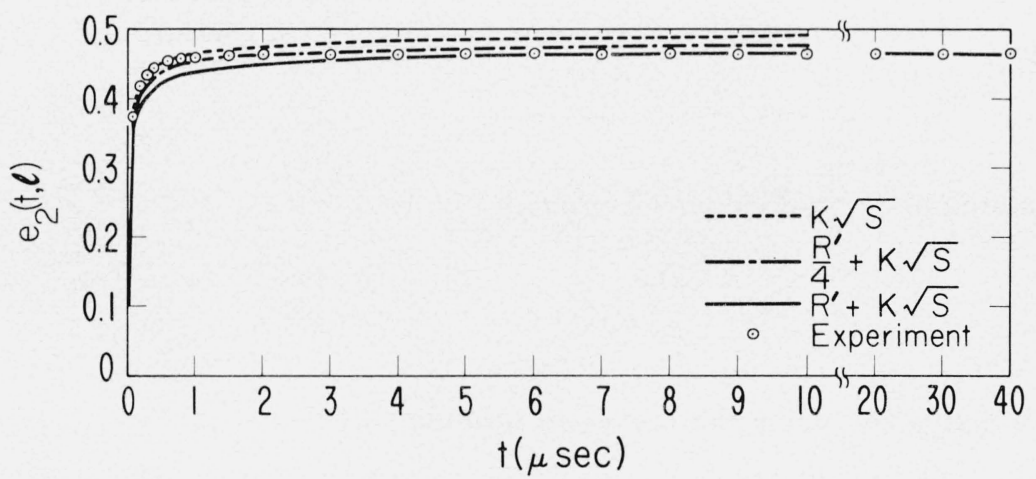

FigurE 3. Step response at receiving end with one unit of time delay $(1 \sqrt{\mathrm{LC}})$ removed.

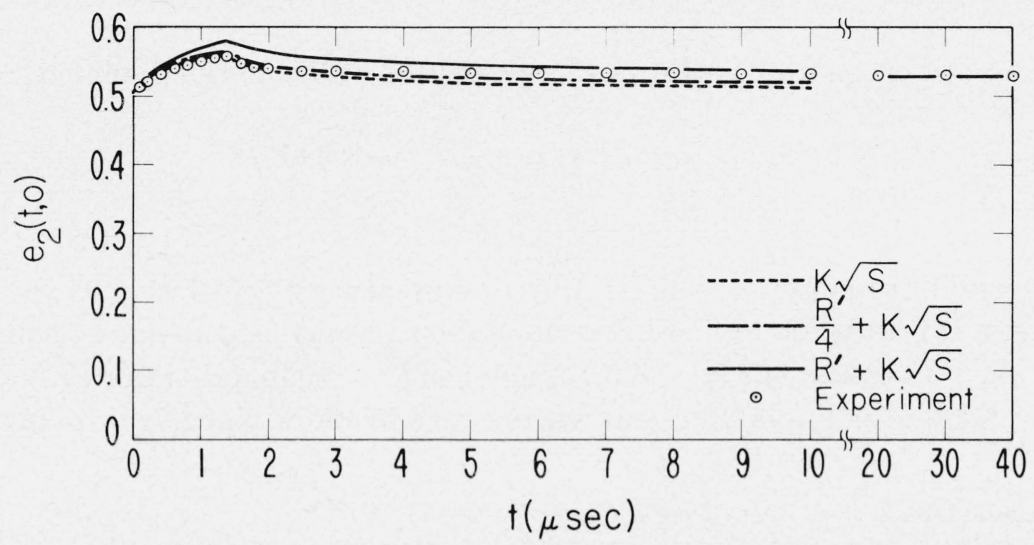

FiguRE 4. Step response at sending end (no time delay). 
Similarly from $(2.5 \mathrm{~b})$

where

$$
D_{n+1,0}^{(1)}=\beta_{0} \frac{n+\frac{1}{2}}{n+1} D_{n, 0}^{(1)}, \quad D_{n, k+1}^{(1)}=\frac{\left(n+k+\frac{1}{2}\right)(n-k)}{(2 k+2)(2 k+3)} \frac{\beta_{2}^{2}}{\beta_{0}} D_{n, k}^{(1)}
$$

$$
D_{n, k}^{(1)}=c_{n+k} d_{n+k+1,2 k+1}^{(1)} \quad(0 \leqslant k \leqslant n \text { and } n=0,1, \ldots) .
$$

In computing $B_{m, n}^{(1)}(x)$ (at $x=0, l$ ) it makes no difference whether (4.5) or (5.5) is implemented to realize $J_{k, m-k}^{(1)}$ since indexing occurs over $k$. However, suppose inquiring only about the magnitude of reflection $n$ occurs. Then coefficients $A_{m, n}^{(1)}$ compute via (4.5) by recurring over the $m$ th now of the $n$th column ${ }^{7}$ whereas (5.5) requires recursion between columns $n$ and $n-1$.

For the time interval ${ }^{8} 0 \leqslant t \leqslant 10 \mu \mathrm{s}$ it is necessary to set $k=1$ (1) 25 and $n=1(1) 10$ to compute the Sheffer polynomials

$$
\sum_{m=0}^{k} A_{k+1-m, n}^{(1)} B_{m, n}^{(1)}(x)
$$

Their ranges of magnitude at $x=0$ and $x=l$ appear below:

$$
\begin{aligned}
& k=1 \quad k=25 \\
& \begin{array}{l|l|l|}
\cline { 2 - 3 } n=0 & f_{0} \times 10^{-2} & f_{1} \times 10^{-14} \\
\cline { 2 - 3 } n=10 & f_{2} \times 10^{-16} & 1<\left|f_{i}\right|<6(i=0, \ldots .3) .
\end{array}
\end{aligned}
$$

Now determining numerical values of erfe $u$

$$
\left(u=\frac{a_{1,1}^{(1)}}{2} \hat{\alpha}_{n}(x) \times\left(\tau-\hat{\alpha}_{n}(x)\right)^{-1 / 2}\right.
$$

through its power series and using the recursion formula [16].

$$
I^{p} \operatorname{erfc} u=-\frac{u}{p} I^{p-1} \operatorname{erfc} u+\frac{1}{2 p} I^{p-2} \operatorname{erfc} u
$$

enables term by term computation of (6.7). In reference to (6.7) let

$$
\hat{e}_{2}(\tau, x)=\sum \hat{e}_{2, n}(\tau, x) \quad \text { and } \quad \hat{e}_{2, n}(\tau, x)=\sum \hat{e}_{2, n, k}(\tau, x)
$$

Then a pair of practical terminating conditions for computing (6.7) can be stipulated:

(a) $\left|\hat{e}_{2, m, q(m)}(\tau, x)\right| \doteq 10^{-5}, \quad m+q(m) \leqslant 25, \quad m=1(1) p$;

(b) $\left|\hat{e}_{2, p}(\tau, x)\right| \doteq 10^{-4}, \quad p \leqslant 10$.

To clarify these conditions suppose that $\hat{e}_{2}(\tau, 0)$ is wanted for $\tau=14.1(t=10 \mu \mathrm{s})$. Reference to (6.7) shows that $n=1(1) 14$ while numerical computation reveals $\hat{e}_{2, m, q(m)}(\tau, 0)$ satisfies condition (a) for $m=1$ (1)4. At $p=4$ conditions (a) and (b) are met and all computations on (6.7) are terminated.

A practical value for the interval of convergence may be determined by invoking the inequality

\footnotetext{
${ }^{7}$ Note the subscript position reversal of $A_{m, n}^{(1)}$ in comparing $G_{n, m}^{(1)}$ of (4.5).

${ }^{*}$ Setting $k=1(1) 50$ and $n=1(1) 10$ jumps computing time on the CDC 3800 from $30 \mathrm{~s}$ to 3 min. This happens because so many convolutions occur. However, it is not necessary to go beyond $k=25$ for this particular transmission line.
} 


$$
2^{k}\left(\tau-\hat{\alpha}_{n}(x)\right)^{k / 2} \quad I^{k} \operatorname{erfc}\left(\frac{a_{1,1}^{(1)}}{2} \hat{\alpha}_{n}(x)\left(\tau-\hat{\alpha}_{n}(x)\right)^{-1 / 2}\right) \leqslant 2^{k} \tau^{\frac{k}{2}} \frac{1}{\Gamma\left(\frac{k}{2}+1\right)}
$$

Substituting the asymptotic expression $\Gamma(z) \sim 2 \pi \epsilon^{-z} z^{z-1 / 2}, k=25$, and computing that value of $\tau$ which satisfies the equation

$$
\tau=\{\Gamma(25 / 2+1)\}^{2 / 25} S_{4}^{-1}
$$

produces $t \doteq 7.4 \times 10^{-6}$ seconds. Now $\left.\hat{e}_{2,1, q(1)}(\tau, x)\right|_{x=0, l}$ does not satisfy condition (a) at $t=2.1 \times 10^{-5} \mathrm{~s}$. Hence, (7.2.7) gives a pessimistic (but useful) guideline for the interval of convergence.

In asymptotic calculations terms corresponding to $N_{0, k}(x)$ and $N_{1, k}(x)$ are dominant and no more then three terms of each series (odd and even) are needed for this combination of parameter values. Asymptotic results are used for $t=20,30,40 \mu \mathrm{s}$.

\section{Appendix A. Bound for $|\sigma|^{1 / 2}$}

We have as a condition from the binomial series (2.1)

$$
\left|\beta_{0}+\beta_{2} \sigma^{1 / 2}\right|<|\sigma| \cdot
$$

Let $|\sigma|=v^{2}$ and invoke the triangular inequality to obtain ${ }^{9}$

$$
v^{2}-\left|\beta_{0}+\beta_{2} \sigma^{1 / 2}\right| \geqslant v^{2}-\beta_{2} v-\beta_{0}=f(v) .
$$

Calculating the positive zero of $f(v)$ yields

$$
v_{0}=\frac{\beta_{2}+\sqrt{\beta_{2}^{2}+4 \beta_{0}}}{2}
$$

It remains to show that $f(v) \geqslant 0$ for $v \geqslant v_{0}$. Noting $f(v)$ has one extremum, a minimum, at $v=\frac{\beta_{2}}{2}$ and $f^{\prime}(v) \geqslant 0$ for $v \geqslant \beta_{2} / 2$ completes the argument.

This bound is sharp since the selection $\sigma^{1 / 2}=v_{0}$ is an admissible value. Transforming from $\sigma$ to $s$ produces the result

$$
S_{2}=\left\{\frac{K}{2 L}+\sqrt{\frac{K}{4 L^{2}}+\frac{R}{L}}\right\}^{2}
$$

\section{Appendix B. Asymptotic Expansion Discussion}

The problem is to show that

$$
\begin{aligned}
e_{2}(t, l) \sim E \operatorname{erfc}\left(\delta t^{-1 / 2}\right)+ & \frac{E}{\pi^{1 / 2}} \exp \left\{-\delta^{2} t^{-1}\right\}\left\{\sum_{N=0}^{\infty} b_{2 n+1,1}^{(2)}\right. \\
& \left.\times \frac{H_{2 n}\left(\delta t^{-1 / 2}\right)}{2^{2 n} t^{\frac{2 n+1}{2}}}+b_{2 n+2,1}^{(2)}+b_{2 n+2,1} \frac{H_{2 n+1}\left(\delta t^{-1 / 2}\right)}{2^{2 n+1} t^{n+1}}\right\} \quad(t \rightarrow \infty)
\end{aligned}
$$

${ }^{9}$ The author is indebted to O. N. Strand for this argument. 
is completely asymptotic $\left(\delta=\frac{l}{2} \sqrt{R C}\right)$.

Let $\varphi_{n}(t) \equiv H_{n-1}\left(\delta t^{-1 / 2}\right) / t^{n / 2}$ and define a sequence $\left\{\psi_{n}(t)\right\}_{n}$ such that

$$
\lim _{t \rightarrow \infty} \frac{\psi_{n+1}(t)}{\psi_{n}(t)}=0 \quad \text { for every } n
$$

Then (B2) forms an asymptotic sequence [17] uniformly in $n$. Using the definition of Hermite polynomials [18] observe the limit quotient

$$
\lim _{t \rightarrow \infty}\left|\frac{\varphi_{2 n+1}(t)}{\varphi_{2 n}(t)}\right|=\lim _{t \rightarrow \infty}\left|\frac{t^{-\frac{2 n+1}{2}}}{t^{-\frac{2 n+1}{2}}}\right| \frac{2}{\delta}=\frac{2}{\delta}
$$

as $t \rightarrow \infty$. Hence, a rearrangement is necessary to satisfy (B2).

It is quite easy to see that odd and even subsequences satisfy respectively

$$
\lim _{t \rightarrow \infty}\left|\frac{\varphi_{2 n+3}(t)}{\varphi_{2 n+1}(t)}\right|=\lim _{t \rightarrow \infty}\left|\frac{\varphi_{2 n+2}(t)}{\varphi_{2 n}(t)}\right|=\lim _{t \rightarrow \infty} \mathrm{O}\left(\frac{1}{t}\right)=0 .
$$

A complete asymptotic expansion with respect to $\left\{\psi_{k}(t)\right\}_{k}$ obeys the relation [19]

$$
f(t)-\sum_{k=0}^{n-1} \psi_{k}(t)=\mathrm{O}\left(\psi_{n}(t)\right), \quad t \rightarrow \infty
$$

By definition of $\left\{\varphi_{2 n}(t)\right\}_{2 n},\left|\varphi_{2 N}(t)\right| \geqslant\left|\varphi_{2 n}(t)\right|(n=N, N+1, \ldots)$ as $t \rightarrow \infty$. Also $b_{2 n+1,1}^{(2)}$ origioriginates from a convergent series, i.e., $b_{2 n+1,1}^{(2)} \leqslant M<\infty$. We have

$$
\left|\sum_{n=N}^{\infty} b_{2 n+1,1}^{(2)} \frac{\varphi_{2 n}(t)}{2^{2 n}}\right| \leqslant M\left|\varphi_{2 N}(t)\right| \sum_{n=N}^{\infty} \frac{1}{2^{2 n}}=\mathrm{O}\left(\varphi_{2 N}(t)\right)
$$

as $t \rightarrow \infty$, a relation which satisfies (B5). Clearly (6) also holds for the odd numbered terms. This argument extends to (6.15) and (6.17) in view of the fact $N_{n, k}(x)$ is uniformly bounded.

The author is sincerely grateful to Prof. N. S. Nahman for his guidance and inspiration throughout the course of this project.

\section{References}

[1] Wigington, R. L., and Nahman, N. S., Transient analysis of coaxial cables considering skin effect, Proc. IRE, 45, 166-174 (Feb. 1957).

[2] Magnuson, P. C., Transient wavefronts on lossy transmission lines-effect of source resistance, IEEE Trans. on Circuit Theory, CT-15, 290-292 (Sept. 1968).

[3] Pelissier, R., The propagation of transient and periodic waves aolng electric lines, Parts I, II, and III, Gen. Rev. of Electricity, 59, pp. 379-399, Sept. 1950, pp. 437-454, Oct. 1950, and pp. 502-512, Nov. 1950 (in French).

[4] Dutta Roy, S. C., and Shenoi, B. A., Distributed and Lumped RC Realization of Constant Argument Impedance, J. Franklin Inst., 282, No. 5, 318-329 (Nov. 1966).

[5] Ho, I. T., and Mullick, S. K., Analysis of transmission lines on integrated-circuit chips, IEEE J. Solid-State Circuits SC-2, No. 4, 201-208 (Dec. 1967).

[6] Nahman, N. S., and Holt, D. R., Time domain reflection and transmission in coaxial cables considering skin effects, to be published.

[7] Titchmarsh, E. C., The Theory of Functions, p. 168 (Oxford Univ. Press, London, England, 2d Edition, 1939). 
[8] Abranowitz, M., and Stegum, I. A., (editors), Handbook of Mathematical Functions with Formulas, Graphs, and Mathematical Tables, National Bureau of Standards Applied Math. Series, 55, 1026 U.S. Gov't Printing Office, Washington, D. C., June 1964).

[9] Op. Cit. [8], p. 299.

[10] Boas, R. P., and Buck, R. C., Polynomial Expansions of Analytic Functions, p. 18 (Springer-Verlag, Berlin, Germany, 1958).

[11] Knopp, K., Theory and Application of Infinite Series, p. 180 and Ch. 12 (Hafner Publishing Co., N.Y., N.Y., 1949).

[12] Gradshteyn, I. S., and Ryzhik, I. M., Tables of Integrals, Series, and Products, p. 14 (Academic Press, N. Y., N. Y., 1965) (translated from Russian).

[13] Doetsch, G., Guide to the Application of Laplace Transforms, p. 198 (D. Van Nostrand, N. Y., N. Y., 1961).

[14] Op. cit. [13], pp. 208, 209.

[15] Stannard, G. E., Calculation of power on a transmission line, Proc. IEEE, 55, No. 1, 132 (jan. 1967).

[16] Loc. cit. [9].

[17] Erdélyi, A., Asymptotic Expansions, p. 8 (Dover Publications, Inc., N. Y., N. Y.).

[18] Op. cit. [8], p. 775.

[19] Op. cit. [17], p. 13.

(Paper 74B3-327) 